\title{
Modeling and measurement of precision reducers' efficiency curve
}

\author{
Hang $\mathrm{Xu}^{1,2, *}$, Jinfeng $\mathrm{Gao}^{1}$, Yuanchun $\mathrm{He}^{3}$, Guiping $\mathrm{Xie}^{3}$, and Yaoting $\mathrm{Wu}^{3}$ \\ ${ }^{1}$ School of Electrical Engineering, Zhengzhou University, Zhengzhou, PR China \\ 2 School of Mechatronics Engineering, Zhongyuan University of Technology, Zhengzhou, PR China \\ ${ }^{3}$ Zhejiang Xiasha Precision Manufacturing Co., Ltd, Ningbo, PR China
}

Received: 8 April 2021 / Accepted: 11 October 2021

\begin{abstract}
Transmission efficiency is the key index when concerning characterizing the performance of precision reducers, and it is usually required to be measured and evaluated. Apart from that, the efficiency value at the rated torque in the efficiency curve is taken as the evaluation value of the efficiency characteristics of precision reducers. However, at present, the research on the efficiency characteristics of precision reducers mainly focuses on theoretical analysis, the development of measurement devices and the measurement methods, while the analysis model of the efficiency curve has not yet been established. In this context, from the perspective of transmission efficiency measurement and evaluation of precision reducers, the efficiency calculation model of precision reducers is established in this paper. Besides, based on the essential change law of the efficiency curve, the efficiency curve analysis model is also set up. To be specific, taking a certain type of RV (rotate vector) reducer as an example, the actual measurement experiments are carried out, and the efficiency curves at different speeds are obtained. The results show that the efficiency of the precision reducer is affected by the measured speed and load torque, and has a positive correlation with the load torque and a negative correlation with the speed. Then, it is proved that the efficiency curve model proposed in this paper can accurately describe the change law followed by the efficiency curve of precision reducers, thus laying a theoretical foundation for the efficiency measurement and evaluation of precision reducers.
\end{abstract}

Keywords: : Precision reducers / RV reducer / efficiency curve / efficiency measurement / logistic function

\section{Introduction}

A precision reducer has high positioning accuracy and repetitive positioning accuracy, and is widely used in robotics, aerospace and other fields. Based on different meshing pairs, the precision reducer can fall into the cycloid precision reducer and the non-cycloid precision reducer [1]. To be specific, the former includes the RV (rotate vector) and the Twinspin reducer, while the latter involves the harmonic reducer and the precision planetary reducer. Among them, the RV reducer is featured with the higher rigidity and is mostly applied to the parts of heavy loads such as the base, arm and shoulder, whereas the harmonic reducer is usually adopted in the forearm, wrist and hand, and the precision planetary reducer is mainly used in Cartesian coordinate robots [2].

Transmission efficiency is an important index for characterizing the performance of precision reducers. Manufacturers of precision reducers usually need to provide the efficiency curve of their products in the sample

\footnotetext{
* Corresponding author: xuhangzzti@126.com
}

manual, and the efficiency value at the rated torque in the efficiency curve is always taken as the evaluation value of the efficiency characteristics of precision reducers [3]. As for the efficiency curve of the precision reducer, it refers to the change curve of efficiency with output torque that is the efficiency-torque curve [4]. Some scholars have carried out researches on the efficiency characteristics of precision reducers, mainly focusing on the theoretical efficiency analysis of precision reducers, the development of measurement devices, the measurement methods and so on. For example, Wang established a theoretical efficiency calculation model of the beveloid gear RV reducer, analyzed the influencing factors of efficiency and optimized the beveloid gear RV reducer with the goal of improving transmission efficiency [5]. Pan built the efficiency calculation model of the filtering gear reducer and conducted efficiency measurement experiments [6]. Wang analyzed the effect of planetary gear/star gear on the transmission efficiency [7-9]. Zhang developed a transmission efficiency measurement system of the cycloid envelope precision reducer [4]. Shi developed a RV reducer comprehensive performance tester, thus being able to realize the comprehensive performance measurement of the 
RV reducer, such as transmission error, stiffness and efficiency [10]. Guan introduced a RV reducer efficiency measurement platform, and used the orthogonal experiment method to carry out the efficiency measurement experiments [11]. Ding made dynamic efficiency measurement of RV reducers and then analyzed the influence of measurement speed and load on efficiency [12]. Han designed an efficiency measurement system for the harmonic reducer [13].

In general, the measurement and evaluation of precision reducer efficiency are closely related to the measurement speed and load torque. At present, the researches mainly focus on the theoretical analysis of efficiency, the development of measurement devices, and the realization of measurement methods. Whereas, the analysis model concerning the efficiency curve of precision reducers has not been established yet.

In this paper, from the perspective of the transmission efficiency measurement and evaluation of precision reducers, the efficiency calculation model and the efficiency curve analysis model are established through the analysis on the efficiency characteristics of precision reducers. Apart from that, the actual measurement experiments are performed. Besides, the correctness of the efficiency curve model is verified through measurement experiments. In this case, the research results in this paper provide a reference for the efficiency measurement and evaluation of precision reducers.

\section{Analysis and modeling of precision reducers' efficiency curve}

\subsection{Efficiency calculation model of precision reducers}

The efficiency of precision reducers is the ratio of the output power to input power, which can be expressed as follows.

$$
\eta=\frac{P_{\text {out }}}{P_{\text {in }}} \times 100 \%=\frac{T_{\text {out }} \times n_{\text {out }}}{T_{\text {in }} \times n_{\text {in }}} \times 100 \%
$$

where $P_{\text {out }}$ and $P_{\text {in }}$ are respectively the output power and input power. $T_{\text {out }}$ and $T_{i n}$ are the output torque and the input torque accordingly, while $n_{\text {out }}$ and $n_{\text {in }}$ are the output speed and input speed separately.

The ratio of the speed at the input shaft of precision reducers to the speed at the output shaft is the transmission ratio $i$, as shown in formula (2).

$$
i=\frac{n_{\text {in }}}{n_{\text {out }}}
$$

Substituting formula (2) into formula (1), the efficiency calculation model of precision reducers can be obtained as follows.

$$
\eta=\frac{T_{\text {out }}}{i \times T_{\text {in }}} \times 100 \%
$$

As a precision reducer receives different forces at different meshing positions during the movement, the torque at the input shaft and the output shaft changes periodically within one cycle of the output shaft. In order to obtain the one-to-one correspondence between efficiency and output torque, the average value of torque is usually employed to calculate the efficiency of precision reducers, as in formula (4) [3].

$$
\eta=\frac{\bar{T}_{\text {out }}}{i * \bar{T}_{i n}} \times 100 \%
$$

where $\bar{T}_{\text {out }}$ is the average value of output torque, and $T_{\text {in }}$ is the average value of input torque.

From the efficiency calculation model of precision reducers, it can be seen that the efficiency is proportional to the ratio of its output torque to the input torque. Therefore, in the transmission efficiency measurement and evaluation of a precision reducer, the transmission efficiency can be obtained by measuring the input torque and output torque of a precision reducer.

\subsection{Modeling of precision reducers' efficiency curve}

The efficiency characteristics of different precision reducers are different from each other, and those of the same precision reducer are also different under different measurement conditions, which is mainly caused by the internal structure of precision reducers, load torque, measurement speed and temperature. Therefore, the efficiency influencing factors of precision reducers can be divided into internal factors and external factors, when the former mainly includes the structure, material and lubrication of precision reducers, while the latter mainly contains the load torque, measurement speed and temperature of precision reducers. In terms of the efficiency measurement and evaluation of precision reducers, it is usually carried out under a constant temperature or thermal equilibrium. Thus, the temperature has little effect. However, the efficiency characteristics are mainly affected by the load torque and the measurement speed, and the efficiency curves of a precision reducer under different measurement conditions are shown in Figure 1 [3].

At a certain speed, the efficiency increases with the increase of the output torque. In the initial stage, when the output torque is low, the efficiency increases with the output torque. Then, as the output torque increases further, the efficiency increases slower and begins to become saturated. Actually, logistic function is a kind of sigmoid function. At first, it grows roughly and exponentially; then as it becomes saturated, the increase slows down, and finally the increase stops when it reaches saturation. The classic Logistic function is shown in formula (5) [14].

$$
P(x)=\frac{K P_{0} e^{r x}}{K+P_{0}\left(e^{r x}-1\right)}
$$

where $K$ is the final value; $P_{0}$ is the initial value; $r$ determines how fast the curve changes, and $x$ is the input variable. 


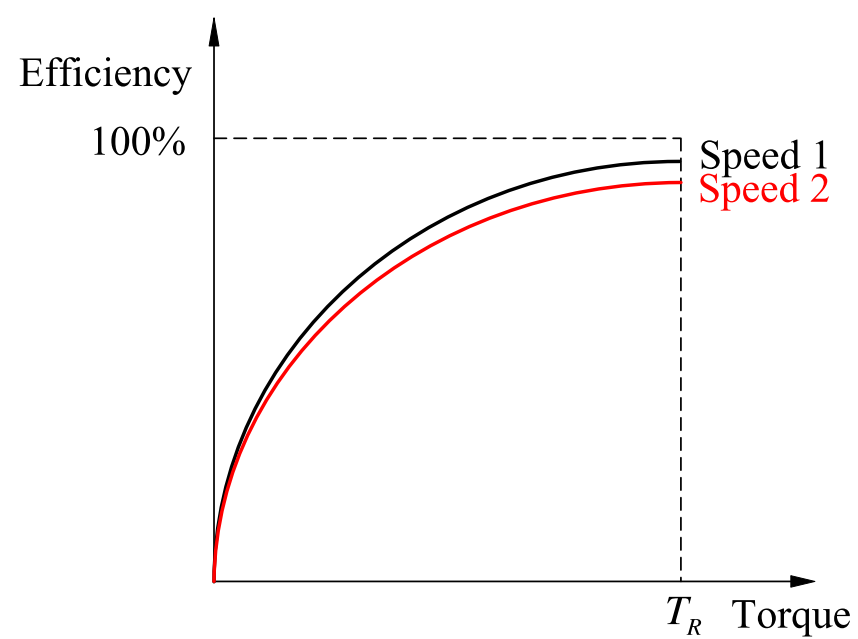

Fig. 1. Efficiency-torque curve.

In this paper, following the essential change law of the efficiency curve of precision reducers, the efficiency curve model of precision reducers is established based on the improved Logistic function, as shown in formula (6)

$$
\eta(T)=\frac{b}{1+a e^{-c T}}-\frac{b}{1+a}
$$

where $a, b$ and $c$ are the coefficients to be measured, while $T$ is the output torque.

The efficiency of precision reducers is also affected by speed. The greater the speed, the lower the efficiency. Besides, the friction torque of precision reducers is proportional to rotational speed. With the increase of rotational speed, the friction torque increases linearly [3]. Furthermore, as the internal friction of precision reducers is the main reason for the loss of efficiency, with the increase of rotational speed, the friction torque increases and the efficiency decreases.

\section{Measurement experiments}

Taking the RV reducer as an example, based on the RV reducer comprehensive performance tester developed by our research group, the efficiency measurement experiments are carried out. The speed ratio of the measured RV reducer is 121 and the rated torque is $784 \mathrm{~N} \cdot \mathrm{m}$.

Two group experiments are designed to measure and analyze the efficiency characteristics of the precision reducer. The first group experiments are conducted on the condition of setting speed and different loads to analyze the influence of torque on the efficiency of the precision reducer. Then, on the basis of the first group experiments, the second group experiments are carried out under different speeds to identify the speed influence on the efficiency of the precision reducer.

\subsection{Measurement device}

The RV reducer comprehensive performance tester developed by our research group is shown in Figure 2 $[2,11]$. In the measurement of transmission efficiency, the



Fig. 2. RV reducer comprehensive performance tester $[2,11]$.

$\mathrm{RV}$ reducer is installed on the precision support and the input shaft is connected with input components (including motors and torque sensors) via precision coupling and driven by a servo motor. In addition, the output flange shaft is connected with output components (including torque sensors and magnetic powder brakes) via precision coupling. Furthermore, the servo motor is controlled by the measurement and control software to work in the speed mode for the purpose of ensuring stable speed during measurement, while the output shaft is loaded by the magnetic powder brake and the load torque is adjusted by a programmable power supply. Beyond that, the angle measurement of input and output shafts is achieved with the high precision circular grating and the resolution can reach $2.06^{\prime \prime}$ and $1.13^{\prime \prime}$. Besides, the dualchannel torque sensor with the precision of $0.1 \% \mathrm{~F} \cdot \mathrm{S}$ is adopted in torque measurements, while the whole system is controlled by the industrial control computer equipped with measurement software to realize the automatic measurement of the comprehensive performance indices belonging to the $\mathrm{RV}$ reducer $[2,15]$.

\subsection{Acquisition and processing of torque signal}

In the efficiency measurement, the servo motor is controlled to work in the speed mode so as to ensure stable speed. The measurement speed (at output shaft) is set to $0.314 \mathrm{rad} / \mathrm{s}$. Within one circle of the output shaft, the measurement torque signals at the input and output shaft of precision reducers change periodically, as shown in Figures 3 and 4 respectively. In order to filter out the interference of noise and obtain the one-to-one correspondence between efficiency and torque, the torque signal is processed with the average filtering method. The average input torque of the precision reducer is $3.0 \mathrm{~N} \cdot \mathrm{m}$, and the average output torque is 318.3 $\mathrm{N} \cdot \mathrm{m}$. According to formula (4), the efficiency of the precision reducer on this measurement condition is $87.69 \%$.

\subsection{Efficiency curve measurement}

On the basis of Section 3.2, the current of the magnetic powder brake is adjusted through the program-controlled power supply, and then the output load is adjusted. 


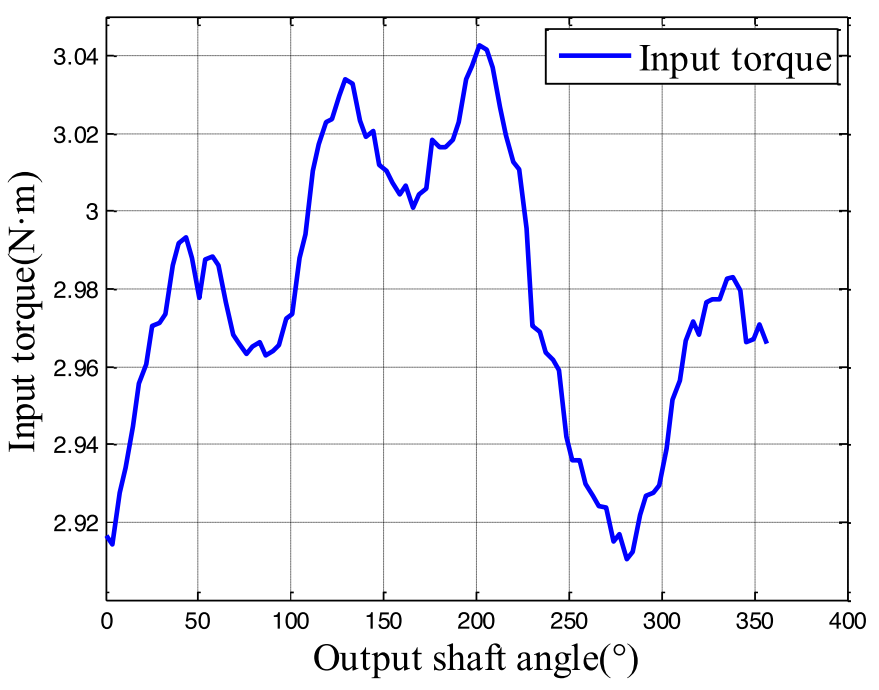

Fig. 3. Input torque $(0.314 \mathrm{rad} / \mathrm{s})$.

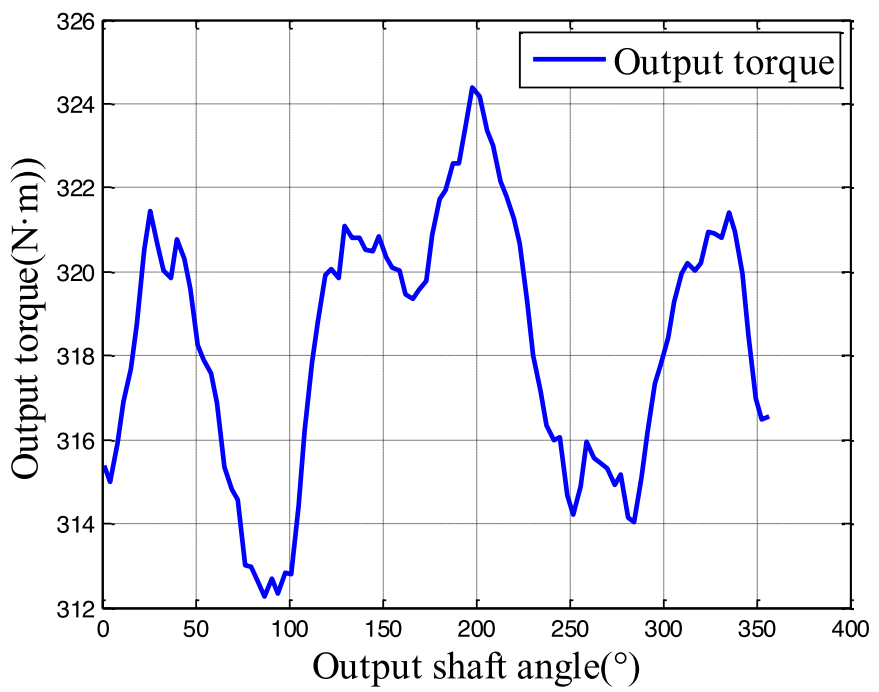

Fig. 4. Output torque $(0.314 \mathrm{rad} / \mathrm{s})$.

By changing the load torque at the output shaft, the efficiency values under different loads can be measured, as shown in Figure 5. Then, based on the actual measurement data, the least square method is adopted to fit the efficiency curve. The fitted efficiency curve is shown in Figure 5. It can be seen that the efficiency is positively correlated with the output torque. In the lower stage of the output torque, the efficiency increases obviously, and with the further increase of the output torque, the efficiency increases slowly and tends to be stable.

The parameters of the efficiency curve model obtained by fitting are shown in Table 1. The value of SSE (Sum of squares due to error) is 0.00065, while that of RMSE (Root mean squared error) is 0.009021, and the value Adj. $R$-Square is 0.9991, indicating that the ideal fitting effect is

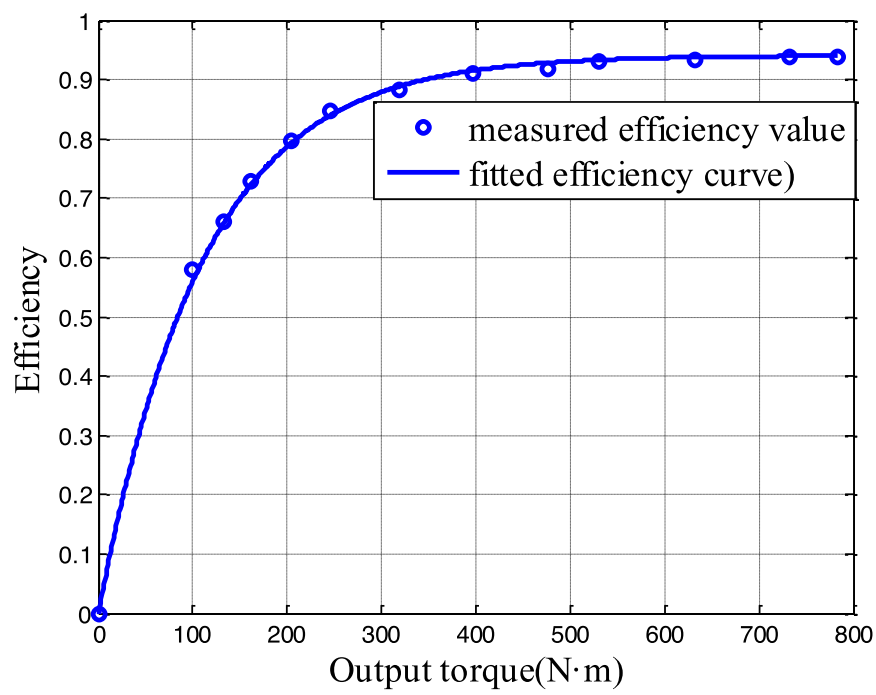

Fig. 5. Efficiency curve $(0.314 \mathrm{rad} / \mathrm{s})$.

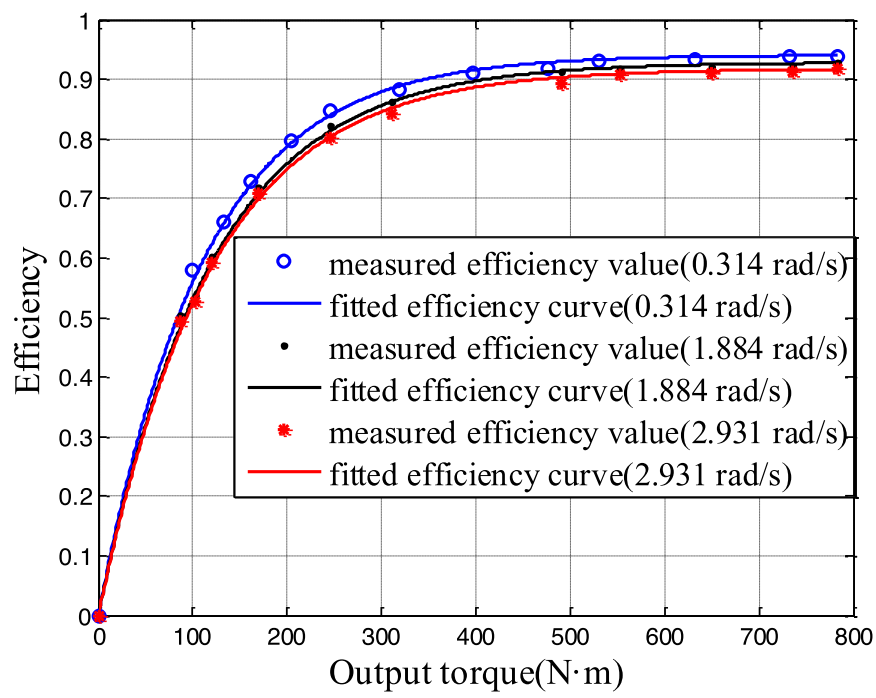

Fig. 6. Efficiency curves at different speeds.

achieved. Therefore, the efficiency curve model established in this paper can accurately describe the efficiency curve change law of precision reducers.

According to the fitting results of the efficiency curve, the rated load $784 \mathrm{~N} \cdot \mathrm{m}$ is substituted into formula (6), and the efficiency value of the reducer under the measurement condition of $0.314 \mathrm{rad} / \mathrm{s}$ and rated load $784 \mathrm{~N} \cdot \mathrm{m}$ is calculated to be $94.00 \%$.

\subsection{Efficiency curves at different speeds}

On the basis of Section 3.3, the efficiency curves at different speeds are obtained by changing the measurement speed, as shown in Figure 6.

The fitting parameters of the efficiency curve model under the measurement condition of $1.884 \mathrm{rad} / \mathrm{s}$ and $2.931 \mathrm{rad} / \mathrm{s}$ are shown in Tables 2 and 3 respectively. 
Table 1. Efficiency curve model fitting parameters.

\begin{tabular}{lllllll}
\hline Parameters & $a$ & $b$ & $c$ & SSE & RMSE & Adj. $R$-Square \\
\hline Value & 0.009993 & 95.08 & 0.0091 & 0.000651 & 0.009021 & 0.9991 \\
\hline
\end{tabular}

Table 2. Fitting parameters of efficiency curve model $(1.884 \mathrm{rad} / \mathrm{s})$.

\begin{tabular}{lllllll}
\hline Parameters & $a$ & $b$ & $c$ & SSE & RMSE & Adj. R-Square \\
\hline Value & 0.004869 & 191.6 & 0.0085 & 0.000643 & 0.009003 & 0.9991 \\
\hline
\end{tabular}

Table 3. Fitting parameters of efficiency curve model $(2.931 \mathrm{rad} / \mathrm{s})$.

\begin{tabular}{lllllll}
\hline Parameters & $a$ & $b$ & $c$ & SSE & RMSE & Adj. $R$-Square \\
\hline Value & 0.009783 & 94.71 & -0.0085 & 0.000632 & 0.009003 & 0.9992 \\
\hline
\end{tabular}

Then, it can be found that the efficiency is negatively correlated with the speed, or in other words, the efficiency decreases with the increase of the speed. Besides, the effect of the output torque on the efficiency is greater than that of the speed on the efficiency.

\section{Discussion}

In the face of achieved experimental results, the following issues can be posed for discussion.

- The efficiency of the precision reducer is proportional to the ratio of its output torque to the input torque. Therefore, the transmission efficiency can be obtained by measuring the input torque and output torque of a precision reducer.

- It can be seen from Figures 2 and 3 that within one cycle of the output shaft, the input and output torque of the precision reducer changes periodically, mainly resulting from different forces at different meshing positions inside the precision reducer. Therefore, within one cycle of the output shaft, the average filtering method can be used to process the torque signal, which can not only obtain the one-to-one correspondence between efficiency and torque, but also can reduce the influence of noise.

- As shown in Figure 4, the efficiency increases with the output torque in the initial stage. Then, as the output torque increases further, the efficiency increases slower and begins to become saturated. Hence, the measurement should cover the process from low torque to high torque as much as possible to objectively reflect the change law of the efficiency curve.

- In this paper, the one-variable function model of efficiency and torque is established. It can make the measurement and evaluation of the efficiency characteristics of precision reducers. However, it should be noted that as the efficiency of precision reducers is related to the speed and load torque, it will be of great significance to establish the two-variable function model of the efficiency curve related to the speed and load.

\section{Conclusions}

Based on the analysis of efficiency characteristics of precision reducers, the efficiency calculation model and the efficiency curve model are set up. Taking the RV reducer as an example, the following conclusions can be obtained.

- The efficiency of precision reducers is affected by measurement speed and load torque. To be specific, efficiency is positively correlated with the load torque and negatively correlated with speed.

- In the lower stage of output torque, the efficiency increases with the increase of torque, and the increasing trend is obvious. With the further increase of torque, the efficiency increases slowly and tends to be saturated.

- Through actual measurement experiments, the efficiency of the measured RV reducer is $94.00 \%$, which verifies the practical effect of the scheme and efficiency curve model proposed in this paper.

- The efficiency curve model put forward in this paper can accurately describe the efficiency curve changing law of precision reducers, and is suitable for the efficiency measurement and evaluation of various precision reducers.

Acknowledgments. The work was supported by the National Natural Science Foundation of China (No. 52075561) and the Key Scientific Research of Henan Higher School (No. 21A460035).

\section{References}

1. L.X. Xu, Y.H. Yang, Dynamic modeling and contact analysis of a cycloid-pin gear mechanism with a turning arm cylindrical roller bearing, Mech. Mach. Theory 104, 327$349(2016)$

2. H. Xu, Z.Y. Shi, B. Yu, H. Wang, Dynamic measurement of the lost motion of precision reducers in robots and the determination of optimal measurement speed, J. Adv. Mech. Des. Syst. Manufactur. 13, 18-00534 (2019) 
3. GB/T 35089-2018, Precision gear transmission for robot-test method (Standardization Administration of the People's Republic of China: Beijing, China, 2018)

4. X. Zhang, Research on test system of transmission efficiency of cycloid enveloping precision reducer, Master degree, Chongqing University, 2015

5. X. Wang, Q.J. Huang, B. Chen, Y.F. Hou, Theoretical analysis of the efficiency of $\mathrm{RV}$ reducer with beveloid gear, $\mathrm{J}$. Mech. Transmiss. 39, 35-38 (2015)

6. J.S. Pan, X.H. Liang, G.J. Wang, Efficiency analysis and experiment of a new type of filtering gear reducer, J. Mech. Transmiss. (05):88-90+119 (2007)

7. C. Wang, The effect of planetary gear/star gear on the transmission efficiency of closed differential double helical gear train, J. Proc. Inst. Mech. Eng. C 234, 4215-4223 (2020)

8. C. Wang, High power density design for planetary gear transmission system, J. Proc. Inst. Mech. Eng. C 233, 56475658 (2019)

9. C. Wang, K. Mao, Design of high power density for RV reducer, J. Br. Soc. Mech. Sci. Eng. 43, 291 (2021)
10. Z.Y. Shi, H. Xu, J.C. Lin, Research on measurement and evaluation of precision reducer lost motion, China J. Sci. Instrum. 39, 56-63 (2018)

11. T. Guan, H.H. Xu, X.W. Xie, L.H. Wei, Transmission efficiency test and analysis of high-precision $\mathrm{RV}$ reducer, J. Mech. Transmiss. 43, 106-109 (2019)

12. C. Ding, S.J. Qin, Test and research on the transmission efficiency of RV reducer, J. Baoji Univ. Arts Sci. (Natural Science Edition) 39, 65-68 (2019)

13. M. Han, K. Liu, K.H. Wu, L. Xu, Study on efficiency test system of harmonic gear box, Mach. Tool Hydraulics 38, 90$92(2010)$

14. J.H. Guan, Y.C. Pei, J.T. Wu, A driving strategy of shape memory alloy wires with electric resistance modeled by logistic function for power consumption reduction, Mech. Syst. Signal Process. 160 (2021)

15. H. Xu, Z.Y. Shi, B. Yu, H. Wang, Optimal measurement speed and its determination method in the transmission precision evaluation of precision reducers, Appl. Sci. Basel $\mathbf{9}$, 0-2146 (2019)

Cite this article as: Hang Xu, Jinfeng Gao, Yuanchun He, Guiping Xie, Yaoting Wu, Modeling and measurement of precision reducers' efficiency curve, Int. J. Metrol. Qual. Eng. 12, 25 (2021) 